\title{
Mode and Intensity of Physical Activity During the Postacute Phase of Sport-Related Concussion: A Systematic Review
}

\author{
Barbara Baker, Eric Koch, Kevin Vicari, and Kyle Walenta
}

\begin{abstract}
Introduction: Sports-related concussions (SRCs) have received attention due to their prevalence in youth. An SRC results from a strong force causing neurological impairment. Recent research has recommended rehabilitation within the first week post-SRC after 24 to 48 hours of rest. The postacute phase is defined as 48 hours to 7 days post-SRC. It is imperative to evaluate the most effective mode and intensity of physical activity to reduce symptoms and improve outcomes. Methods: CINAHL, PubMed, SPORTDiscus, and Web of Science databases were used to search the terms "brain concussion" AND "exercise" and variations of these terms. The evidence level for each study was evaluated using the 2011 Oxford Center for Evidence-Based Medicine Guide. The methodological rigor of each study was evaluated using a scale adapted from Medlicott and Harris. Results: Two thousand sixty-eight records were identified. Six studies were included in this systematic review. Three studies were classified as moderately strong. The remaining 3 studies were considered weak. Five of the studies used either a cycle ergometer or a treadmill. The sixth study used walking, cycling, and swimming, as well as sports drills. All of these modes of exercise were determined to be safe. All studies utilized low- and moderate-intensity interventions, which were found to be nondetrimental and showed improved recovery time and symptom resolution. Five of the studies also incorporated components of high-intensity exercise that was also found to be nondetrimental, and they showed a positive influence on recovery time and symptom resolution. However, all activity in each of the reviewed studies started at a low level and progressed up to a higher level only as each individual client's symptoms permitted. Discussion: Overall, this review found that various modes of activity at light-, moderate-, and highintensity levels are efficacious and can be safely used during the postacute phase of SRC. Conclusion: Though the volume of literature at this time is limited, therapists should consider prescribing closely monitored individualized exercise programs utilizing progressive intensities when treating patients during the postacute phase of SRC.
\end{abstract}

Keywords: exercise, sports-physiotherapist, brain-concussion, systematic reviews/meta-analyses/critically appraised topics, research designs/area(s)

The media has shed light on the risks and consequences of sport-related concussions (SRCs). Increased attention has led to an abundance of new research developments on the most effective rehabilitation measures following an SRC. According to the Center for Disease Control's report of traumatic brain injuries diagnosed in an emergency department, the incidence rate for traumatic brain injuries in patients aged 15-24 years was double that for patients aged 24-44 years. ${ }^{1}$ One of the hallmarks of a concussion is the presence of neurological signs and symptoms that occur as a result of an external force to the brain, even though the concussion does not produce any macroscopic damage to neural tissue. ${ }^{2}$ In these acute injuries, there is an immediate release of neurotransmitters along with ion fluxes that lead to increased neuronal depolarization. ${ }^{3}$ The depolarization increases the workload of the sodiumpotassium pump, requiring increased energy consumption, and an eventual adenosine triphosphate. ${ }^{3}$ The lack of energy is what is thought to put the brain in a vulnerable state during the acute recovery phase postconcussion and, thus, why it is so important to explore the most accurate, evidence-based recommendations for the safe return to play. Current research establishes the postacute recovery phase following a concussion as 24 to 48 hours after the trauma occurred. ${ }^{4}$

The symptoms of SRCs can manifest in many different domains. ${ }^{2}$ Some of the symptoms that are most specifically reported are headaches, dizziness, impaired balance, drowsiness, loss

The authors are with Grand Valley State University, Grand Rapids, MI, USA. Baker (bakerb@gvsu.edu) is corresponding author. of memory, and concentration problems. ${ }^{5-7}$ It is common for individuals to experience a spontaneous resolution of symptoms within 2 to 3 weeks; therefore, until recently, the consensus regarding traditional treatment included physical and cognitive rest until the symptoms were resolved. ${ }^{8-10}$

McCrea et $\mathrm{al}^{11}$ studied 635 concussed collegiate and high school athletes and concluded that a symptom-free waiting period that was used in $60 \%$ of their cases did not influence clinical recovery or reduce the risk of another concussion. Yet, there was continued high utilization of waiting periods until the symptomfree state was reached. Pfaller et al ${ }^{12}$ found a $99.3 \%$ prescription rate of rest following SRCs from 2012 to 2014 while following 143 athletes. It must be remembered that this was determined to be best practice at that time. Recent studies have contradicted the idea that complete rest facilitates optimal recovery. Meehan and Bachur ${ }^{10}$ completed a study that found that participants who were prescribed strict rest had a slower symptom resolution and had a higher symptom burden during the first 10 days after their emergency department visit than those prescribed usual care. Thomas et $\mathrm{al}^{13}$ found that adolescents who received usual care, including 1 to 2 days of rest (acute phase) followed by a gradual increase in nonsymptom-producing activities during the postacute period, had fewer symptoms over 10 days compared with the intervention group prescribed strict rest. This was further emphasized by Buckley et al, ${ }^{14}$ who found that acutely concussed athletes 18 21 years old who received no postinjury accommodations returned to an asymptomatic status sooner than those who were prescribed strict rest. 
Recently, the paradigm of postconcussion activity recommendations has shifted. Schneider et $\mathrm{al}^{4}$ found that a brief period of cognitive and physical rest is appropriate for most patients (24-48 h), followed by a postacute period (48 h until symptom free) in which patients should be encouraged to engage in gradual progressive physical activity. There is insufficient evidence that complete rest will achieve the most successful recovery in the most efficient time frame. ${ }^{9}$ While 24 to 48 hours of rest postinjury is recommended, patients can be encouraged to begin cognitive and physical activity that remains below the threshold of symptom exacerbation following this time period. ${ }^{9}$ Lempke et al's ${ }^{15}$ systematic review, which supports this recommendation, concluded that physical activity performed within 0 to 7 days postconcussion resulted in a $32 \%$ decline in multiple reported symptoms when compared with those who were prescribed strict rest. The authors also noted that the physical activity group experienced greater symptom resolution at day 7 , while the rest group reported greater symptom severity at day 10 .

Most athletes recover gradually within a few days following an SRC, but up to $15 \%$ have reported not returning to play for at least 30 days. ${ }^{16}$ This indicates that there may be an opportunity to improve concussion rehabilitation for patients with SRCs, as not all recover within a few days. As treatment focus shifts toward earlier physical exertion, it is imperative to evaluate the most effective mode and intensity of the physical activity to reduce symptoms and improve outcomes during this postacute phase. While strict rest may delay or hinder recovery, it has also been reported that overly strenuous physical and cognitive exertion during this time frame can exacerbate symptoms, decrease neurocognitive performance, and increase the risk of secondary injury. ${ }^{17}$ Consequently, data regarding mode and intensity of this postacute exercise could help shape future rehabilitation techniques for patients following an SRC.

The purpose of this systematic review was (1) to investigate the most effective mode of physical activity during the postacute phase of SRC and (2) to assess the most effective intensity of exercise during the postacute phase of SRC.

\section{Methods}

\section{Databases and Search Terms}

The CINAHL, PubMed, SPORTDiscus, and Web of Science databases were used to search the terms "brain concussion" AND "exercise" and variations of these terms. The MeSH terms utilized in the PubMed search were "brain concussion," "exercise," "physical exertion," and "exercise tolerance." The terms were reviewed with an expert librarian to ensure the most complete search. The literature search was conducted in January of 2019. Following database searches, duplicate articles were removed, and the remaining articles were screened based on the title of the article and abstract. In order to rule articles "in" or "out" with optimal thoroughness, 3 separate assessors each evaluated two thirds of the total number of articles by title. If there were any disagreements about an article between 2 of the assessors, the third assessor served as the tiebreaker. The 3 assessors then screened the remaining articles by abstract with the same tie-break format. After the 2 screens, the included articles were evaluated by a full-text assessment to determine their eligibility for this review by all 3 assessors. A PRISMA flow diagram was utilized to display the search process (Figure 1). ${ }^{18}$

\section{Inclusion and Exclusion Criteria}

The following inclusion criteria were used in this systematic review: (1) original research (consisting of randomized controlled trials, retrospective studies, case studies; level 2, 3, or 4), (2) participants younger than the age of 25 years, (3) concussion as the primary source of injury, (4) an intervention with specified intensity or mode of exercise following a concussion, and (5) at least one group initiating exercise on average within 7 days following concussion. The following exclusion criteria were used in this systematic review: (1) participants older than 25 years; (2) expert opinion and clinical commentary, level 5; (3) studies not written in English.

\section{Evidence Level}

The Oxford Center for Evidence-Based Medicine 2011 Levels of Evidence was used to establish the evidence level of each study included in this systematic review (Table 1). ${ }^{19}$ The included studies were individually assessed by 3 authors to reduce the risk of bias. If the authors disagreed, a common conclusion was reached via discussion.

\section{Methodological Rigor}

Methodological rigor was evaluated using the methods described by Medlicott and Harris (Table 2). ${ }^{20}$ The included studies were assessed by 3 authors to the reduce risk of bias. If the authors disagreed, a discussion occurred to reach a common conclusion.

\section{Results}

\section{Search Strategy}

Four electronic databases revealed 2068 articles. One additional article was discovered when the researchers identified an abstract of interest and contacted the author for a completed copy of the study. The author, Dr Leddy, informed the researchers that the abstract they had found was currently only an abstract. However, he did direct them to a new publication that was appropriate to be screened. Duplicates were eliminated, and 1315 articles were screened by title, as displayed in the PRISMA 2009 flow diagram. ${ }^{18}$ Of these 1315 article titles, 1160 were excluded based on the title, which resulted in 155 abstracts. About 155 abstracts were screened, and 133 articles were excluded following the abstract review, which resulted in 22 full-text articles to be assessed for eligibility based on the inclusion and exclusion criteria. Six studies were found to be eligible and were used in this systematic review.

\section{Evidence Level}

Based on the 2011 Oxford Center for Evidence-Based Medicine Guide, ${ }^{19} 3$ articles $^{21-23}$ used in this systematic review were considered level 2 evidence because they were randomized controlled trials. The remaining 3 articles ${ }^{22,24,25}$ were considered level 3 evidence, as they consisted of prospective cohort studies, retrospective cohort studies, and nonrandomized controlled cohort studies (Table 3).

\section{Methodological Rigor}

Methodological rigor was evaluated using the methods described by Medlicott and Harris (Table 4). ${ }^{20}$ Two studies ${ }^{21,24}$ were scored as $6 / 10$, three studies ${ }^{22,23,26}$ were scored as $4 / 10$, and one study ${ }^{27}$ 
2068 records identified through database searching
One additional record identified through other sources

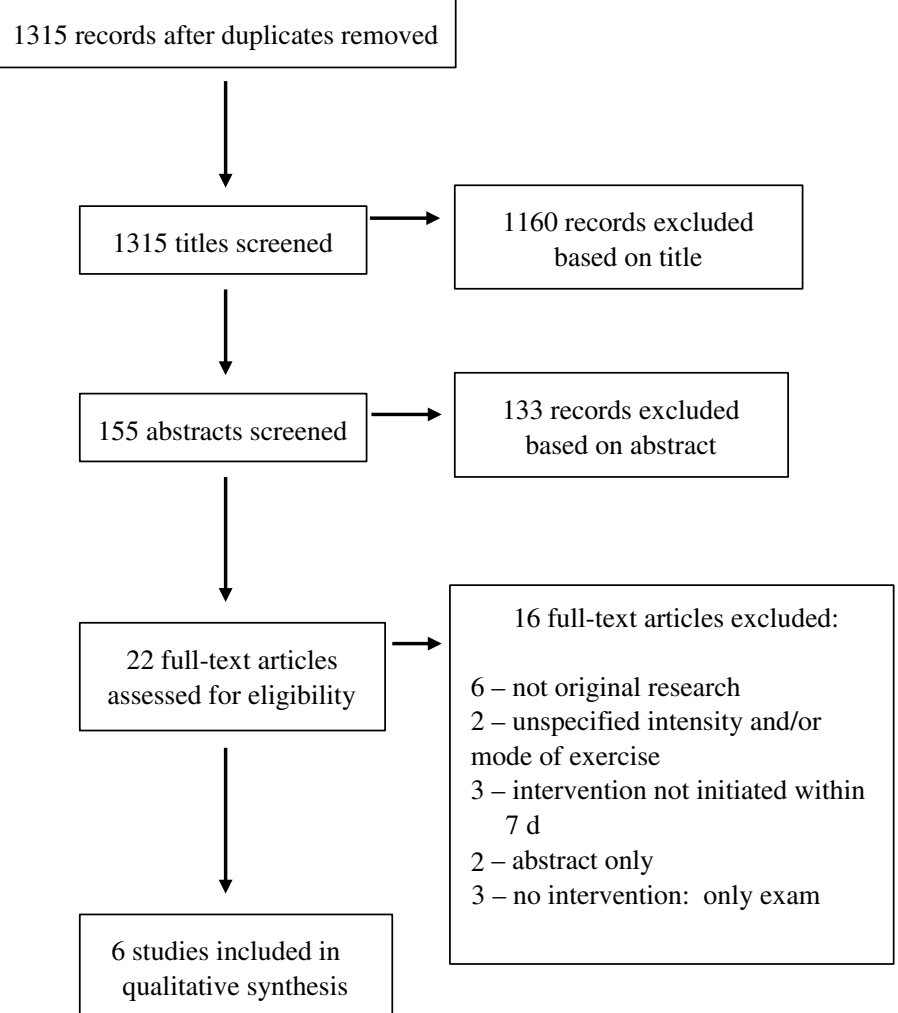

Figure 1 - PRISMA 2009 flow diagram. ${ }^{18}$

Table 1 Evidence Level Overview ${ }^{14}$

\begin{tabular}{ll}
\hline Hierarchy & Evidence level criteria \\
\hline 1 & $\begin{array}{l}\text { Systemic reviews of randomized trials or n-of-1 } \\
\text { trials } \\
\text { Randomized trials or observational studies with } \\
\text { dramatic effect } \\
\text { Nonrandomized controlled cohort or follow-up } \\
\text { studies } \\
\text { Case series, case-control studies, or historically } \\
\text { controlled studies } \\
\text { Mechanism-based reasoning }\end{array}$ \\
\hline
\end{tabular}

was scored as 3/10. Detailed information on the specific rigor rating characteristics of each included study can be found in Table 2.

\section{Summary of Studies}

Grool et $\mathrm{al}^{26}$ enrolled 3063 participants who presented to the emergency department with an acute head injury. In total, 2413 participants, ages 5-18 years, diagnosed with a concussion completed the study. The purpose was to investigate the association between participation in physical activity within 7 days postinjury and the incidence of persistent postconcussive symptoms (PPCS). The patients initiated physical activity within 48 hours of receiving
Table 2 Medlicott and Harris Methodological Rigor

\begin{tabular}{ll}
\hline Item & Rigor criteria \\
\hline 1 & Randomization \\
2 & Inclusion and exclusion criteria listed \\
3 & Similarity of groups at baseline \\
4 & Treatment protocol was sufficiently described to be \\
& replicable \\
5 & Reliability of data obtained with the outcome mea- \\
& sures was investigated \\
6 & Validity of data obtained with the outcome measures \\
7 & was investigated \\
8 & Blinding of patient, treatment provider, and assessor \\
9 & Dropouts were reported \\
10 & Long-term results were assessed via follow-up \\
\hline & Adherence to home programs was investigated \\
\hline
\end{tabular}

a diagnosis of concussion. The participants then completed a survey at 7 and 28 days postinjury. The parameters of each subcategory of physical activity and exercise intensity are outlined in Table 5. In total, about $70 \%$ of the subjects participated in physical activity by 7 days postenrollment and were assessed for PPCS at 28 days postinjury. The light-aerobic group had a $31.4 \%$ risk of developing PPCS, compared with the no-physical-activity 
Table 3 Evidence Level Results ${ }^{19}$

\begin{tabular}{|c|c|c|}
\hline Study & Hierarchy & Evidence level score \\
\hline Grool et $\mathrm{al}^{26}$ & Prospective cohort study & 3 \\
\hline Lawrence et $\mathrm{al}^{27}$ & Retrospective cohort study & 3 \\
\hline Leddy et $\mathrm{al}^{21}$ prelim & Retrospective comparative trial & 3 \\
\hline Leddy et $\mathrm{al}^{24}$ & Randomized controlled trial & 2 \\
\hline Maerlender et $\mathrm{al}^{22}$ & Randomized controlled trial & 2 \\
\hline Micay et $\mathrm{al}^{23}$ & Randomized controlled trial & 2 \\
\hline
\end{tabular}

Table 4 Methodological Rigor Results ${ }^{20}$

\begin{tabular}{|c|c|c|c|c|c|c|c|c|c|c|c|}
\hline Study & Item 1 & Item 2 & Item 3 & Item 4 & Item 5 & Item 6 & Item 7 & Item 8 & Item 9 & Item 10 & $\begin{array}{l}\text { Methodological } \\
\text { rigor score }\end{array}$ \\
\hline Grool et $\mathrm{al}^{26}$ & $\mathrm{~N}$ & $\mathrm{Y}$ & $\mathrm{N}$ & $\mathrm{Y}$ & $\mathrm{N}$ & $\mathrm{Y}$ & $\mathrm{N}$ & $\mathrm{Y}$ & $\mathrm{N}$ & $\mathrm{N}$ & $4 / 10$ \\
\hline Lawrence et $\mathrm{al}^{27}$ & $\mathrm{~N}$ & $\mathrm{Y}$ & $\mathrm{N}$ & $\mathrm{Y}$ & $\mathrm{N}$ & $\mathrm{N}$ & $\mathrm{Y}$ & $\mathrm{N}$ & $\mathrm{N}$ & $\mathrm{N}$ & $3 / 10$ \\
\hline Leddy et $\mathrm{al}^{21}$ prelim & $\mathrm{N}$ & Y & $\mathrm{Y}$ & $\mathrm{Y}$ & $\mathrm{Y}$ & $\mathrm{Y}$ & $\mathrm{N}$ & $\mathrm{Y}$ & $\mathrm{N}$ & $\mathrm{N}$ & $6 / 10$ \\
\hline Leddy et $\mathrm{al}^{24}$ & $\mathrm{Y}$ & Y & $\mathrm{Y}$ & $\mathrm{Y}$ & $\mathrm{Y}$ & $\mathrm{N}$ & $\mathrm{N}$ & $\mathrm{Y}$ & $\mathrm{N}$ & $\mathrm{Y}$ & $7 / 10$ \\
\hline Maerlender et $\mathrm{al}^{22}$ & $\mathrm{Y}$ & $\mathrm{N}$ & $\mathrm{Y}$ & $\mathrm{Y}$ & $\mathrm{N}$ & $\mathrm{N}$ & $\mathrm{N}$ & $\mathrm{Y}$ & $\mathrm{N}$ & $\mathrm{N}$ & $4 / 10$ \\
\hline Micay et $\mathrm{al}^{23}$ & $\mathrm{Y}$ & $\mathrm{N}$ & $\mathrm{Y}$ & $\mathrm{Y}$ & $\mathrm{N}$ & $\mathrm{N}$ & $\mathrm{N}$ & $\mathrm{Y}$ & $\mathrm{N}$ & $\mathrm{N}$ & $4 / 10$ \\
\hline
\end{tabular}

Note: 1 . Randomization; 2. Inclusion and exclusion criteria listed; 3 . Similarity of groups at baseline; 4 . Treatment protocol was sufficiently described to be replicable; 5 . Reliability of data obtained with the outcome measures was investigated; 6 . Validity of data obtained with the outcome measures was investigated; 7. Blinding of patient, treatment provider, and assessor; 8. Dropouts were reported; 9. Long-term results were assessed via follow-up; 10. Adherence to home programs was investigated.

group, who had a risk of $43.5 \%$. The risk for developing PPCS decreased with increased physical activity intensity, as the moderate-exercise group had a risk of $24.4 \%$ and the high-intensity group had a $14.5 \%$ risk of developing PPCS.

Lawrence et $\mathrm{al}^{27}$ completed a study that retrospectively analyzed 253 subjects, all of whom had sustained acute concussions and were 15-20 years old. The purpose of the study was to determine whether earlier time to initiation of aerobic exercise (AE) following acute concussion is associated with time to full return to sport, school, and work. Each patient was prescribed a daily standardized stationary bike protocol containing 4 levels, as follows: (1) 15 minutes of cycling at a heart rate (HR) of 100 to 120 beats per minute (bpm), (2) 30 minutes at 100 to $120 \mathrm{bpm}$, (3) 30 minutes at $140 \mathrm{bpm}$, and (4) intervals of 1-minute maximal sprints every 5 minutes, totaling 30 minutes. The results determined that a shorter time to initiation of $\mathrm{AE}$ postconcussion was associated with a faster full return to sport, school, and work.

A study performed by Leddy et $\mathrm{al}^{21}$ examined 54 adolescents with acute SRCs. The purpose was to analyze the effect of early prescribed $\mathrm{AE}$ compared with relative rest on the rate of recovery. The relative rest group was composed of 30 subjects from a previous Leddy et al study ${ }^{28}$ that received recommendations based on usual care postconcussion. The exercise group consisted of 24 subjects who were prescribed a subthreshold AE program. The programs were individualized per participant based on $80 \%$ of the participants' HR threshold on the Buffalo Concussion Treadmill Test (BCTT). The subjects were instructed to engage in supervised, daily treadmill or stationary bike exercise at their subthreshold HR for 20 minutes or until symptoms were exacerbated. The recovery time was significantly faster for the exercise group compared with the rest group (8.29 vs $23.93 \mathrm{~d}$ ). By day 14, the exercise group also had significantly fewer participants who remained symptomatic. Finally, no participants from the exercise group had delayed recovery, whereas 4 rest group participants had delayed recovery.
Leddy et $\mathrm{al}^{24}$ followed 103 subjects, 13-18 years old, within 10 days following an SRC. The subjects were randomized into an $\mathrm{AE}$ intervention group and a palcebo-like stretching group. On average, activity was initiated within 5.0 days for both groups. The intervention group was instructed to perform $\mathrm{AE}$ each day on a stationary bike or treadmill at a prescribed target HR. The prescription target HR was calculated at $80 \%$ of the HR achieved at symptom exacerbation on the BCTT at the first visit. The participants were instructed to stop their home exercise session if their symptoms increased by 2 or more points (visual analog scale) from their preexercise symptom level or at 20 minutes, whichever came first. A new target HR was determined by weekly clinic BCTT performance for as long as the participant remained symptomatic. The participants in the stretching group were instructed to follow a prescribed whole-body stretching program for 20 minutes per day. The stretching program was advanced each week, much like the AE prescription. The AE participants recovered in a median of 13 days, whereas the stretching group recovered in 17 days. The incidence of participants with delayed recovery was higher in the stretching group $(n=7)$ than the AE group $(n=2)$. Finally, the total symptom scores appeared to decrease more rapidly in the exercise group, although this was not statistically significant.

Maerlender et $\mathrm{al}^{22}$ conducted a study on recently concussed college athletes to determine the effect of daily moderate-intensity stationary cycling on recovery following an SRC. Fifteen concussed athletes were given "standard concussion recommendations," and 13 concussed athletes completed "daily physical exertion at a moderate level." The participants initiated exercise within 2 days following injury. The athletes assigned to the exertion protocol rode a stationary bicycle at a perceived level of exertion of "mild" to "moderate" (reported perceived exertion = $0-6){ }^{25}$ The athletes rode the bike for a total of 20 minutes unless symptom increases became uncomfortable for the athlete. This was performed daily until each athlete achieved clinical recovery. 


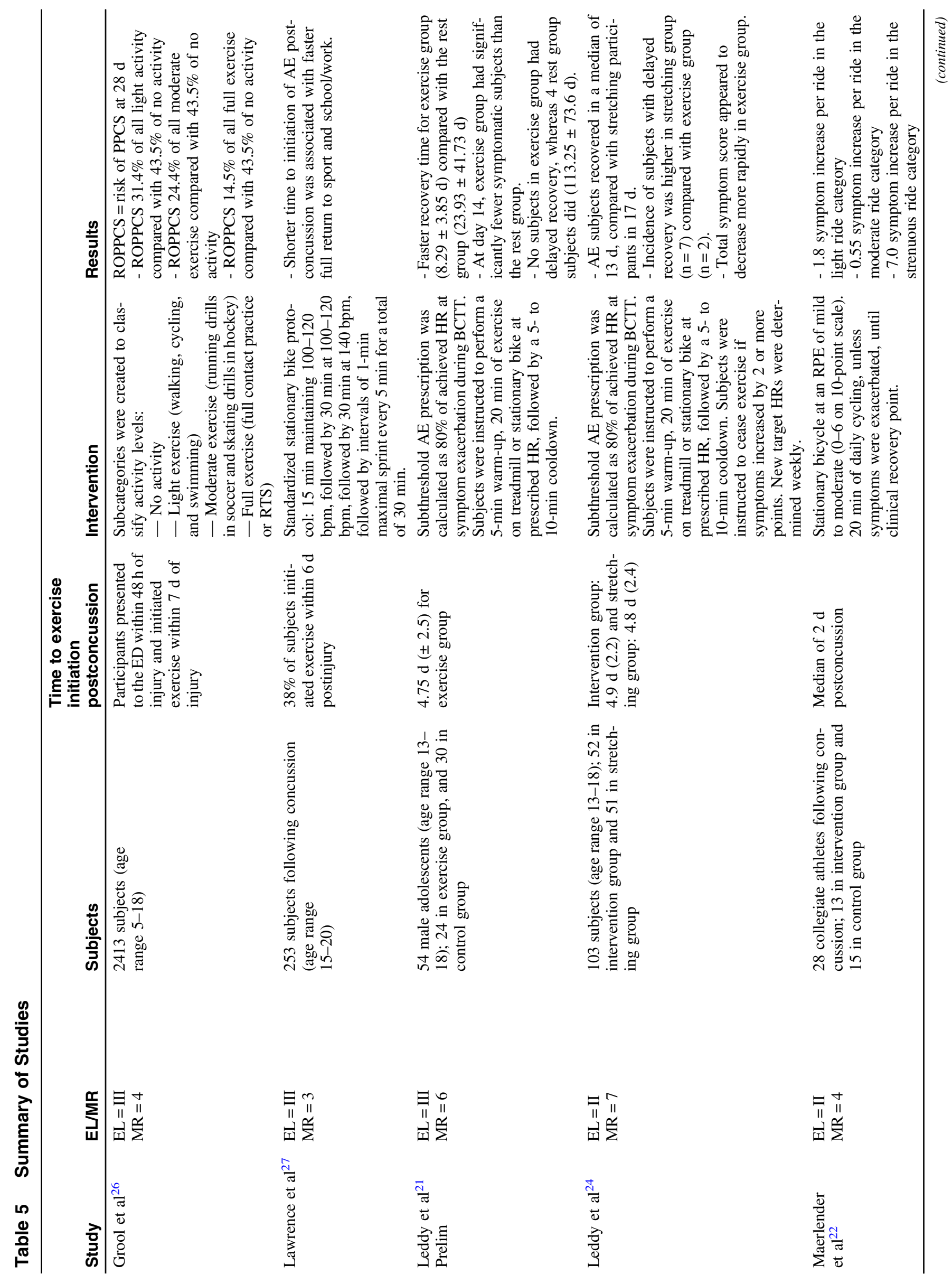




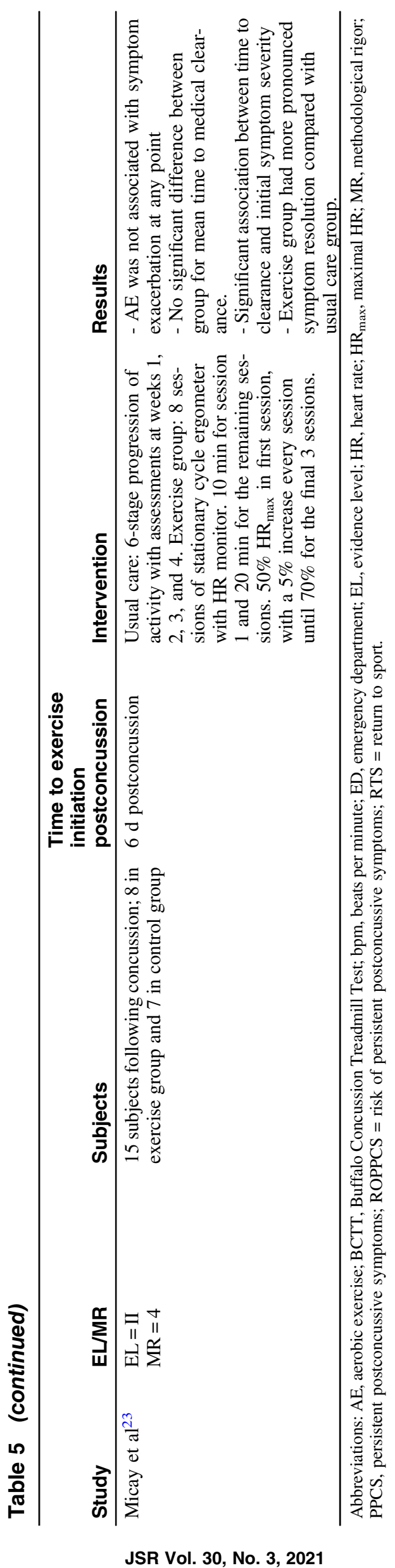


The median number of days to recover was no different by group (exertion group $=15 \mathrm{~d}$ and control group $=13 \mathrm{~d}$ ). A difference in symptom scores between subjects in the exertion group was reported. There was a 1.8 symptom increase per ride rated in "light" rides, a 0.55 symptom increase in "moderate" rides, and a 7 symptom increase per "strenuous" ride.

Micay et $\mathrm{al}^{23}$ analyzed 15 male adolescents who sustained acute SRCs. Exercise was initiated within 5 days post-SRC. The purpose of the study was to examine the feasibility of implementing a standardized AE intervention versus usual care following an SRC. The usual care group was directed through a 6-stage progression of activity and was assessed at weeks 1, 2, 3, and 4. The intervention consisted of 8 sessions on a stationary cycle ergometer while wearing an HR monitor to ensure appropriate exercise intensity. Session 1 consisted of 10 minutes of cycling at a $50 \%$ age-predicted maximal HR $\left(\mathrm{HR}_{\max }\right)$, whereas session 2 was the same intensity but for 20 minutes. All subsequent sessions maintained a duration of 20 minutes and increased the exercise intensity by $5 \%$ of the subjects' age-predicted $\mathrm{HR}_{\text {max }}$ for each session. The final 3 sessions were performed at the subjects' $70 \%$ age-predicted $\mathrm{HR}_{\max }$ for 20 minutes. Post-concussion Symptom Scale (PCSS) scores were collected before and after each session, and exacerbation was defined as an increase in PCSS scores by 3 or more points. The study concluded that AE intervention was not associated with symptom exacerbation at any point during or immediately after the exercise sessions. No significant difference was found between groups for mean time to medical clearance; however, there was a significant association identified between time to medical clearance and initial symptom severity.

A summary of the included studies can be found in Table 5.

\section{Discussion}

The protocols for SRC are changing and are supported by the current literature. ${ }^{4,10,13,14,15}$ Schneider et $\mathrm{al}^{4}$ found that a brief period of cognitive and physical rest is appropriate for most patients (24-48 h), followed by a postacute period ( $48 \mathrm{~h}$ until symptom free) in which patients should be encouraged to engage in gradual progressive physical activity. Although gradual progressive physical activity is now recommended by the literature, no studies have addressed the mode or intensity of that physical activity during the postacute period.

The mode and intensity of the recommended physical activity are the focus of this systematic review. The 6 articles reviewed support the use of stationary cycling in the postacute period. ${ }^{21-24,26,27}$ Leddy's 2 articles ${ }^{21,24}$ also supported the use of a treadmill during the postacute period in SRC. Grool ${ }^{26}$ also used walking and swimming during the postacute period, with no deleterious effect.

The reviewed articles also supported recommendations for intensity of the gradual progressive physical activity recommended by Schneider et al. ${ }^{4}$ All 6 of the reviewed studies supported the use of minimal, as well as moderate, physical activity in the postacute period of SRC. However, each study defined minimal and moderate physical activity in slightly different ways and also progressed the activity in various ways. Grool ${ }^{26}$ defined "light activity" as minimal exertion during walking, cycling, or swimming. Moderate activity was described by $\mathrm{Grool}^{26}$ and included the actual running of drills in sports such as hockey or soccer. Lawrence ${ }^{27}$ started subjects after SRC at an HR of 100 to $120 \mathrm{bpm}$ during light activity. His protocol then increased the subjects to 100 to $120 \mathrm{bpm}$ for 30 minutes, still considered light activity. As tolerated, the subjects were then increased to $140 \mathrm{bpm}$ for 30 minutes for moderate activity. In both of Leddy's studies, ${ }^{21,24}$ he used the HR at which the subjects became symptomatic on the BCTT as a baseline. Then, to determine initial light activity level, they took $80 \%$ of the achieved HR on the BCTT. Each week, the HR achieved on the BCTT was reevaluated. Maerlender ${ }^{22}$ used the Reported Perceived Exertion scale to rate each client's activity level. In this study, each client's reported perceived exertion was kept between 0 and 6 on a 10 -point scale. The final study, performed by Micay, ${ }^{23}$ had subjects begin at a light activity level by prescribing an $\mathrm{HR}$ at $50 \%$ of $\mathrm{HR}_{\max }$ per age. The exercise was progressed to a moderate level by increasing HR by $5 \%$ per session as long as the client remained asymptomatic. These studies ${ }^{21,22,24-27}$ involving moderate-intensity exercise reported no adverse events, demonstrated improved clinical outcomes, and had superior outcomes to low-intensity exercise when comparison was available. The length of recovery was also shortened following light to moderate activity in the postacute phase as compared with subjects with SRC who were told to rest or not to exercise.

Five of the included studies ${ }^{21,23,24,26,27}$ had some utilization of high-intensity exercise incorporated during the postacute phase. Overall, this literature involving high-intensity exercise during the postacute phase of SRC reports no adverse effects with highintensity exercise. It should be noted that the studies that utilized high-intensity exercise did this as part of a protocol that started with light physical activity and progressed slowly, as the symptoms allowed.

Overall, this review found that various modes of activity at light-, moderate-, and high-intensity levels are efficacious and can be safely used during the postacute phase of SRC. However, all activity in each of the reviewed studies started at a low level and progressed up to a higher level only as each individual client's symptoms permitted.

\section{Limitations of the Systematic Review}

This systematic review and the included studies were not without limitations. When investigating concussion interventions and evaluating recovery, it is possible that subjects who recover fastest or have the highest exercise tolerance were those who experienced less severe concussions. Only 1 of the 6 included studies ${ }^{27}$ accounted for concussion severity by grouping the participants by whether or not they missed time participating in their sport. In addition, 4 of the 6 included studies ${ }^{22,23,26,27}$ were rated as having "weak" methodological rigor on the scale developed by Medlicott and Harris. ${ }^{20} \mathrm{~A}$ further limitation of the review is that there are currently no randomized controlled trials explicitly comparing 2 or more modes or 2 or more intensities of exercise during the postacute phase. Furthermore, none of the included studies investigated long-term results via follow-up at 6 months or greater, which challenges the effectiveness of the interventions. Several of these limitations may be attributed to the changing guidelines for concussion treatment during the postacute phase in recent years.

\section{Suggestions for Future Research}

Considering the shifting guidelines for concussion treatment and the limited number of studies that met the inclusion criteria of this review, there are undoubtedly directions for future research. Since various modes and intensities of exercise post-SRC have not been explicitly compared to date, researchers should consider conducting randomized controlled trials comparing different modes of exercise and different exercise intensities for exercise prescription 
during the postacute phase of SRC. Future studies should incorporate concussion severity to ensure that it is not a confounding variable in regard to intervention effectiveness on recovery. Studies have shown that persistent symptoms following a concussion are common and may persist for several months ${ }^{29}$; therefore, future studies involving early exercise initiation should collect and evaluate long-term outcomes. The literature surrounding exercise during the postacute phase of SRC is encouraging, and patients will certainly benefit from further research and improved treatment guidelines.

\section{Conclusion}

SRCs are extremely prevalent. Recent literature has shed light on the benefits of initiating exercise during the postacute phase of SRCs; however, the optimal mode or intensity of exercise during this time was previously not well defined. Nonaversive, as well as positive, results were shown during the postacute phase using various modes of exercise, including cycle ergometry, treadmill, walking, cycling, swimming, and even sports drills. Therefore, therapists should consider an individualized approach to exercise mode prescription during the postacute phase while carefully considering exercise intensity. Though the volume of literature at this time is limited, therapists should consider prescribing closely monitored individualized exercise programs utilizing progressive intensities when treating patients during the postacute phase of SRC.

\section{Acknowledgment}

The authors would like to thank Betsy Williams, MSLIS.

\section{References}

1. Centers for Disease Control. Rates of TBI-related Emergency Department visits by age group-United States, 2001-2010: concussion, traumatic brain injury. CDC Injury Center; 2014. http://www.cdc. gov/traumaticbraininjury/data/rates_ed_byage.html. Accessed May 20, 2018.

2. McCrory P, Meeuwisse WH, Aubry M, et al. Consensus statement on concussion in sport: the 4th international conference on concussion in sport held in Zurich, November 2012. Br J Sports Med. 2013; 47(5):250-258. PubMed ID: 23479479 doi:10.1136/bjsports-2013092313

3. Giza CC, Hovda DA. The neurometabolic cascade of concussion. J Athl Train. 2001;36(3):228-235. PubMed ID: 12937489

4. Schneider KJ, Leddy JJ, Guskiewicz KM, et al. Rest and treatment/ rehabilitation following sport-related concussion: a systematic review. Br J Sports Med. 2017;51(12):930-934. PubMed ID: 28341726 doi:10.1136/bjsports-2016-097475

5. Brett BL, Kuhn AW, Yengo-Kahn AM, et al. Initial symptom presentation after high school football-related concussion varies by time point in a season: an initial investigation. Sports Med Open. 2018;4(1):8. PubMed ID: 29387986 doi:10.1186/s40798-018-0121-8

6. De Kruijk JR, Leffers P, Meerhoff S, Rutten J, Twijnstra A. Effectiveness of bed rest after mild traumatic brain injury: a randomised trial of no versus six days of bed rest. J Neurol Neurosurg Psychiatry. 2002;73(2):167-172. doi:10.1136/jnnp.73.2.167

7. Lau BC, Kontos AP, Collins MW, Mucha A, Lovell MR. Which on-field signs/symptoms predict protracted recovery from sportrelated concussion among high school football players? Am J Sports Med. 2011;39(11):2311-2318. PubMed ID: 21712482 doi:10.1177/ 0363546511410655
8. Pinto SM, Twichell MF, Henry LC. Predictors of pharmacological intervention in adolescents with protracted symptoms after sports-related concussion. PM R. 2017;9(9):847-855. PubMed ID: 28093374 doi:10. 1016/j.pmrj.2016.12.009

9. McCrory P, Meeuwisse W, Dvořák J, et al. Consensus statement on concussion in sport - the 5th international conference on concussion in sport held in Berlin, October 2016. Br J Sports Med. 2017; 51(11):838-847. PubMed ID: 28446457

10. Meehan WP 3rd, Bachur RG. The recommendation for rest following acute concussion. Pediatrics. 2015;135(2):362-363. PubMed ID: 25560439 doi:10.1542/peds.2014-3665

11. McCrea M, Guskiewicz K, Randolph C, et al. Effects of a symptom-free waiting period on clinical outcome and risk of reinjury after sport-related concussion. Neurosurgery. 2009;65(5):876883. PubMed ID: 19834399 doi:10.1227/01.NEU.0000350155. 89800.00

12. Pfaller AY, Nelson LD, Apps JN, Walter KD, McCrea MA. Frequency and outcomes of a symptom-free waiting period after sportrelated concussion. Am J Sports Med. 2016;44(11):2941-2946. PubMed ID: 27371548 doi:10.1177/0363546516651821

13. Thomas DG, Apps JN, Hoffmann RG, McCrea M, Hammeke T. Benefits of strict rest after acute concussion: a randomized controlled trial. Pediatrics. 2015;135(2):213-223. PubMed ID: 25560444 doi:10.1542/peds.2014-0966

14. Buckley TA, Munkasy BA, Clouse BP. Acute cognitive and physical rest may not improve concussion recovery time. J Head Trauma Rehabil. 2016;31(4):233-241. PubMed ID: 26394292 doi:10.1097/ HTR.0000000000000165

15. Lempke L, Jaffri A, Erdman N. The effects of early physical activity compared to early physical rest on concussion symptoms. J Sport Rehabil. 2019;28(1):99-105. PubMed ID: 28952910 doi:10.1123/jsr. 2017-0217

16. Kerr ZY, Zuckerman SL, Wasserman EB, Covassin T, Djoko A, Dompier TP. Concussion symptoms and return to play time in youth, high school, and college American football athletes. JAMA Pediatr. 2016;170(7):647-653. PubMed ID: 27135397 doi:10.1001/ jamapediatrics. 2016.0073

17. Majerske CW, Mihalik JP, Ren D, et al. Concussion in sports: postconcussive activity levels, symptoms, and neurocognitive performance. J Athl Train. 2008;43(3):265-274. PubMed ID: 18523563 doi:10.4085/1062-6050-43.3.265

18. Moher D, Liberati A, Tetzlaff J, Altman DG. Preferred reporting items for systematic reviews and meta-analyses: the PRISMA statement. Ann Intern Med. 2009;151(4):264-269. PubMed ID: 19622511 doi:10.7326/0003-4819-151-4-200908180-00135

19. CEBM. Oxford Centre for Evidence-Based Medicine Levels of Evidence. 2011. http://www.cebm.net/wp-content/uploads/ 2014/06/CEBMLevels-of-Evidence-2.1.pdf. Accessed January 20, 2019.

20. Medlicott MS, Harris SR. A systematic review of the effectiveness of exercise, manual therapy, electrotherapy, relaxation training, and biofeedback in the management of temporomandibular disorder. Phys Ther. 2006;86(7):955-973. PubMed ID: 16813476 doi:10.1093/ptj/ 86.7.955

21. Leddy JJ, Haider MN, Hinds AL, Darling S, Willer BS. A preliminary study of the effect of early aerobic exercise treatment for sport-related concussion in males. Clin J Sport Med. 2018;28(1):13-20. PubMed ID: 29257777 doi:10.1097/JSM.0000000000000431

22. Maerlender A, Rieman W, Lichtenstein J, Condiracci C. Programmed physical exertion in recovery from sports-related concussion: a randomized pilot study. Dev Neuropsychol. 2015;40(5):273-278. PubMed ID: 26230745 doi:10.1080/87565641.2015.1067706 
23. Micay R, Richards D, Hutchison MG. Feasibility of a postacute structured aerobic exercise intervention following sport concussion in symptomatic adolescents: a randomised controlled study. BMJ Open Sport Exerc Med. 2018;4(1):e000404. PubMed ID: 30018795 doi:10. 1136/bmjsem-2018-000404

24. Leddy JJ, Haider MN, Ellis MJ, et al. Early subthreshold aerobic exercise for sportrelated concussion: a randomized clinical trial. JAMA Pediatr. 2019;173(4):319-325. PubMed ID: 30715132 doi:10.1001/jamapediatrics.2018.439

25. Borg G. Borg's Perceived Exertion and Pain Scales. Champaign, IL: Human Kinetics; 1998.

26. Grool AM, Aglipay M, Momoli F, et al. Association between early participation in physical activity following acute concussion and persistent postconcussive symptoms in children and adolescents.
JAMA. 2016;316(23):2504-2514. PubMed ID: 27997652 doi:10. 1001/jama.2016.17396

27. Lawrence DW, Richards D, Comper P, Hutchison MG. Earlier time to aerobic exercise is associated with faster recovery following acute sport concussion. PLoS One. 2018;13(4):e0196062. PubMed ID: 29668716 doi:10.1371/journal.pone.0196062

28. Leddy JJ, Hinds AL, Miecznikowski J, et al. Safety and prognostic utility of provocative exercise testing in acutely concussed adolescents: a randomized trial. Clin J Sport Med. 2018;28(1):1320. PubMed ID: 29257777 doi:10.1097/JSM.0000000000000431

29. Graham R, Institute of Medicine and National Research Council. Sports-Related Concussions in Youth: Improving the Science, Changing the Culture. Washington, DC: National Academies Press; 2014. 